\title{
Fístula broncopleural cutânea causada por Eikenella corrodens
}

\author{
Bronchopleural cutaneous fistula due to Eikenella corrodens \\ Kin-Sun Wong1, Yhu-Chering Huang ${ }^{2}$
}

\section{Resumo}

Objetivo: Fazer uma revisão sobre fístula cutânea broncopleural causada por Eikenella corrodens e relatar e discutir o caso de uma paciente.

Descrição: Paciente do sexo feminino, 16 anos de idade, foi encaminhada ao nosso hospital em estado febril com histórico de escarro com estrias de sangue há 2 semanas; apresentava seqüelas neurológicas de encefalite herpética e estava confinada ao leito desde os cinco anos de idade. A mãe da paciente relatou ter detectado, dias antes, uma massa mole paraespinhal longitudinal. A paciente recebia alimentação via oral apesar da ocorrência de freqüentes engasgamentos nos últimos anos. À apalpação, a massa podia ser pressionada até encontrar menor resistência do espaço subcutâneo, estendendo-se longitudinalmente até a região torácica inferior. A tomografia torácica revelou pneumonia necrotizante do lóbulo direito inferior e uma fístula pleurocutânea levando a áreas de cavitação subcutânea. A paciente foi submetida a tratamento prolongado com antibióticos com decrescentes coletas de ar por 8 semanas.

Comentários: A Eikenella corrodens tem sido cada vez mais indicada como potencial patógeno causador de infecções pleuropulmonares. Não há relatos de fístula pleurocutânea a formação de abscessos como complicadores de empiema e pneumonia necrotizante causada por infecção de $E$. corrodens. Presença de lesão torácica proeminente que aumenta e diminui com a respiração pode ser uma indicação de fístula pleurocutânea. O tratamento do empiema de Eikenella com antibióticos sem decorticação cirúrgica exige um tratamento prolongado com antibioticoterapia.

J Pediatr (Rio J). 2005;81(3):265-7: Fístula cutânea, eikenella, infecção, antibióticos.

\section{Introdução}

A Eikenella corrodens tem sido implicada como um potencial patógeno causador de infecções pleuropulmonares $^{1-3}$. No entanto, fistulas broncopleurais com formação de

1. Mestre. Professor assistente, Departamento de Pediatria, Chang Gung Children's Hospital, Taoyuan, Taiwan.

2. Doutor. Professor, Departamento de Pediatria, Chang Gung Children's Hospital, Taoyuan, Taiwan.

Artigo submetido em 09.11.04, aceito em 17.01.05.

Como citar este artigo: Wong KS, Huang YC. Fístula broncopleural cutânea causada por Eikenella corrodens. J Pediatr (Rio J). 2005;81:265-7.

\section{Abstract}

Objective: The aim of this paper is to review the subject and to report on and discuss a case of bronchopleural cutaneous fistula due to Eikenella corrodens.

Description: A 16-year-old girl was brought to our hospital with fever and blood-tinged sputum 2 weeks prior to her admission. She suffered from neurologic sequelae of herpetic encephalitis and had been bed-ridden since 5 years of age. A longitudinal paraspinal soft mass had been noted in the previous week by her mother. She had been given oral feeding despite frequent choking for the past few years. On palpation, the mass can be squeezed to follow the least resistance of subcutaneous space longitudinally extending to the lower thoracic region. Chest computed tomography scan revealed right lower lobe necrotizing pneumonitis and a pleuro-cutaneous fistula leading to the subcutaneous air locules. A protracted course of antibiotics was prescribed and subcutaneous air trapping decreased in size over 8 weeks.

Comments: Eikenella corrodens has increasingly been implicated as a potential causative pathogen in pleuropulmonary infections. Pleuro-cutaneous fistula and abscess formation complicating empyema and necrotizing pneumonitis due to $E$. corrodens infection have not been reported. A bulging thoracic subcutaneous lesion waxes and wanes with respiration suggest the possibility of a pleruo-cutaneous fistula. Treatment of Eikenella empyema using antibiotics without surgical decortication requires a prolonged course of antibiotic therapy.

J Pediatr (Rio J). 2005;81(3):265-7: Cutaneous fistula, eikenella, infection, antibiotics.

abscessos seguidas de empiema e pneumonite necrotizante (PN) resultantes de infecções por E. corrodens ${ }^{4}$ já foram também relatadas. Este relato apresenta o caso de uma paciente de 16 anos de idade com desnutrição de longa duração e aspirações pulmonares recorrentes, causadas por um abscesso frio paraespinhal resultante de empiema da pleura a fistula broncopleural.

\section{Relato de caso}

Paciente com 16 anos de idade, foi encaminhada ao nosso hospital, em estado febril, apresentando escarro com estrias de sangue há 2 semanas. 
Mãe havia detectado massa paraespinhal, em situação longitudinal, que comprometia região toraco-lombo-sacral, e que aumentava e diminuía durante acessos de tosse. A paciente estava confinada ao leito desde os 5 anos de idade devido a uma encefalite herpética. Recebia alimentação por via oral, apesar de sofrer freqüentes engasgamentos nos últimos anos. Encontrava-se desnutrida, com severa escoliose toraco-lombar e portadora de contratura rígida das extremidades. Durante ausculta torácica foram observadas crepitações bilaterais e murmúrio vesicular diminuído na porção inferior do pulmão direito. A radiografia torácica apresentou infiltrações pulmonares bilaterais nos lobos inferiores e presença de ar loculado à direita em região subpulmonar. Uma tomografia computadorizada (TC) do tórax revelou PN no lobo inferior direito e pneumotórax. Foram aspirados $15 \mathrm{ml}$ de secreção francamente purulenta da massa flutuante paraespinhal. Uma nova TC após a aspiração e inserção de um cateter tipo pigtail mostrou uma fístula broncopleural persistente, levando a formação de ar loculado no subcutâneo (Figura 1).

Um crescimento misto de E. corrodens e Capnocytophagia sputigena foi observado após 5 dias. A família da paciente não autorizou o esvaziamento do empiema e a decorticação pleural; foram então administradas empiricamente clindamicina e gentamicina parenteral. O tratamento com antibióticos foi de longa duração, 8 semanas, com gradual resolução de coletas de ar das coleções aéreas no subcutâneo.

\section{Discussão}

A paciente apresentava paralisia cerebral, e a disfunção na deglutição predispunha-a a freqüentes aspirações e infecções no trato respiratório. Subseqüente disseminação por continuidade da PN e empiema levaram a formação de fístula broncopleural cutânea. Diferente da PN em adultos com prognóstico pobre, os pacientes pediátricos com PN podem ser seguramente tratados com antibióticos, apresentando melhora completa ${ }^{5}$. Intervenções cirúrgicas em casos de PN ficam limitadas a decorticação de pulmão encarcerado, afecção por bactérias anaeróbicas e empiema, não responsivo à drenagem torácica, ou com presença de fístula broncopulmonar persistente. ${ }^{5}$.

A E. corrodens é uma habitante normal da cavidade oral humana, e dos tratos respiratórios e gastrointestinais, e tem se mostrado um patógeno incomum de empiema em pacientes pediátricos ${ }^{3,4}$. Trauma local, presença de doença crônica, neoplasias, cirurgia prévia do intestino, síndrome de Down, paralisia cerebral e retardo mental predispõem as infecções por E. corrodens. Em pacientes adultos, a bactéria $E$. corrodens é freqüentemente encontrada em infecções no pescoço, cabeça, pulmão e intra-abdominais. Joshi et al. relataram 24 casos de infecções pulmonares causadas por E. corrodens, dentre esses, 15 pacientes tiveram efusão pleural mas nenhum apresentou infiltrações nas estruturas adjacentes $^{2}$ Killen et al. foram os primeiros a descreverem um caso de empiema parapneumônico causado por $E$. corrodens com envolvimento do espaço intrapleural, identificado em paciente do sexo masculino, 33 anos de idade ${ }^{4}$.

$A E$. corrodens apresentou um padrão não usual de suscetibilidade ao antibiótico, sendo não suscetível a agentes antimicrobianos normalmente utilizados contra a maioria dos anaeróbios, como a clindamicina e o metronidazol. A drenagem cirúrgica oportuna dos abscessos e o debridamento adequado de tecidos devem ser considerados em casos de infecções progressivas causadas por $E$. corrodens $^{6}$. A lesão torácica subcutânea proeminente, apresentando aumento e diminuição durante a respiração, sugere a possibilidade da presença de uma fístula broncopleural, terapia conservadora com antibióticos e aspiração em tais casos podem demandar um tratamento prolongado com antibióticos.

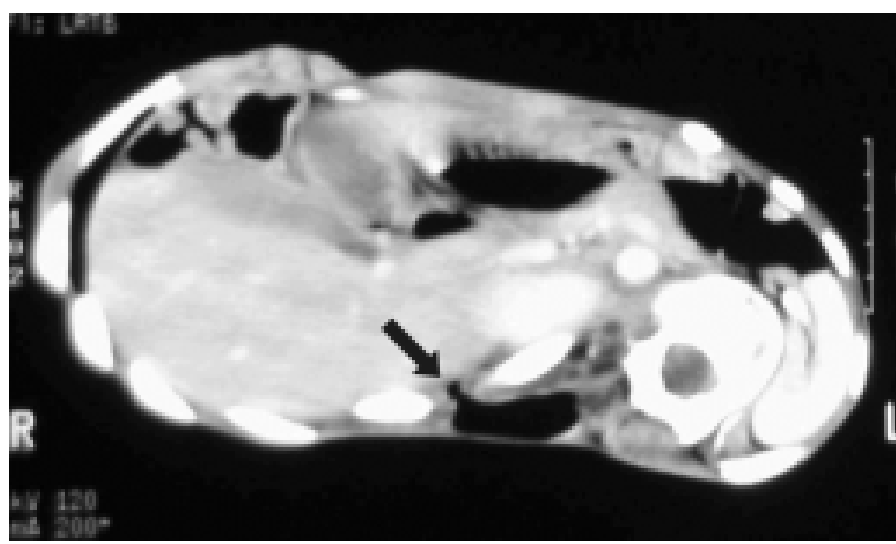

Figura 1 - Tomografia computadorizada do tórax apresentando fístula broncopleural em comunicação com o recesso costofrênico e bolsos de ar na parte de trás (seta) 


\section{Referências}

1. St John MA, Belda AA, Matlow A, Prober CG. Eikenella corrodens empyema in children. Am J Dis Child. 1981;135:415-7.

2. Joshi N, O'Bryan T, Appelbaum PC. Pleuropulmonary infections caused by Eikenella corrodens. Rev Infect Dis. 1991;13:1207-12.

3. Paul K, Patel SS. Eikenella corrodens infections in children and adolescents: case reports and review of literature. CID 2001;33:54-60.

4. Killen JW, Swift GL, White RJ. Pleuropulmonary infection with chest wall infiltration by Eikenella corrodens. Thorax. $1996 ; 51: 871-2$.

5. Wong KS, Chiu CH, Yeow KM, Huang YC, Liu HP, Lin TY. Necrotizing pneumonitis in children. Eur J Pediatr. 2000;159: 684-8.
6. Kosloske AM, Cushing AH, Shuck JM. Early decortication for anaerobic empyema in children. J Pediatr Surg. 1980;15:422-9.
Correspondência:

Kin-sun Wong

Chang Gung Children's Hospital

5 Fu Hsin Street, Taoyuan, Taiwan

E-mail: pchest@adm.cgmh.org.tw

Fone: $886-3-3281200$

Fax: 886-3288957 